\title{
Determinação de metais em águas de abastecimento público: um estudo de caso, município de Ouro Preto/MG
}

Ao longo das últimas quatro décadas a preocupação com a qualidade da água para consumo humano vem ganhando destaque no Brasil, sendo criados e aperfeiçoados marcos legais pertinentes ao tema. O município de Ouro Preto/MG está inserido no Quadrilátero Ferrífero, que em razão de suas características geológicas, bem como atividades ligadas à mineração, possibilitam a ocorrência de determinados metais em seus mananciais hídricos. O serviço de abastecimento de água do município conta com estruturas de tratamento convencional e simplificado, em diferentes localidades. Diante da possibilidade de ocorrência de determinados metais e semimetais nas águas destinadas ao abastecimento público do município, acima do valor máximo permitido pela legislação brasileira, este trabalho buscou realizar a identificação e quantificação de metais por meio da utilização da técnica de Fluorescência de Raio-X por Reflexão Total (TXRF) no LCMEMUFOP. Em relação às vinte amostras de água bruta, três apresentaram resultados acima do máximo permitido para cobre, seis para zinco e manganês, e nove para ferro. Das vinte amostras de água tratada, duas oriundas de sistemas com tratamento simplificado apresentaram resultados acima do valor máximo permitido, uma para ferro e uma para manganês.

Palavras-chave: Água para Consumo Humano; Saúde Pública; Metais; Fluorescência de Raios-X Por Reflexão Total.

\section{Determination of metals in public water supply: a case study, municipality of Ouro Preto/MG}

\begin{abstract}
Over the past four decades the concern with the quality of drinking water has been gaining attention in Brazil, creating and improving legal frameworks relevant to the theme. The city of Ouro Preto, Minas Gerais, Brazil, located in the Iron Quadrangle, which due to its geological characteristics allow the occurrence of certain metals in their water sources. In addition, human activities such as mining, agriculture and domestic and industrial activities can change the water characteristics. The water service of the city account with structures of conventional and simplified treatments, in different localities. Faced with the possibility of occurrence of metals and semimetals in water intended for municipal public supply above the maximum permitted limits stablished by the Brazilian law, this study attempts to make the identification and quantification of metals by total reflection X-ray fluorescence (TXRF) in LCMEM-UFOP. Regarding the twenty samples of raw water, three presented results above the maximum allowed for copper, six for zinc and manganese, and nine for iron. Of the twenty treated water samples, two from systems with simplified treatment presented results above the maximum allowed value, one for iron and one for manganese.
\end{abstract}

Keywords: Drinking Water; Public Health; Metals; Total Reflection X-Ray Fluorescence.

Topic: Engenharia Sanitária

Reviewed anonymously in the process of blind peer
Received: $10 / 04 / 2018$

Approved: 24/05/2018
Alisson Martins Ramos (it)

Universidade Federal de Minas Gerais, Brasil

http://orcid.org/0000-0002-3703-3370

http://lattes.cnpq.br/4180829976307660

amramosbh@gmail.com

Robson José de Cássia Franco Afonso (ii)

Universidade Federal de Ouro Preto, Brasil

http://lattes.cnpq.br/4560322826290409

http://orcid.org/0000-0003-1940-3304

robsonafonso@iceb.ufop.br

\section{Referencing this:}

RAMOS, A. M.; AFONSO, R. J. C. F.. Determinação de metais em águas de abastecimento público: um estudo de caso, município de Ouro Preto/MG. Revista Ibero Americana de Ciências Ambientais, v.9, n.4, p.192-199, 2018. DOI: http://doi.org/10.6008/CBPC2179$\underline{6858.2018 .004 .0016}$ 


\section{INTRODUÇÃO}

Nas últimas décadas, existe uma havido crescente preocupação com a qualidade da água para consumo humano no Brasil. Foram feitos vários esforços para garantir que as pessoas tenham acesso a água de qualidade. Diversos atores sociais, como governo, sociedade civil, empresas e instituições de pesquisa têm trabalhado para melhorar os instrumentos que regulam o uso da água em seus diferentes contextos. A água possui características dinâmicas, que podem ser modificadas por diversos fatores. A variação das condições ambientais pode alterar os metais que estão concentrados nos sedimentos de um rio, permitindo sua dissolução em períodos de chuvas (SANTOS et al., 2008). A qualidade da água resulta de fenômenos naturais e antrópicos, assim, o uso e a ocupação do solo podem interferir diretamente em suas características (VON SPERLING, 2005).

A qualidade da água para consumo humano no Brasil ganhou relevância nas políticas públicas vigentes, relacionadas diretamente à saúde pública e preservação do meio ambiente. Com o esforço de garantir a qualidade da água para consumo humano no Brasil, houve uma grande evolução nos marcos legais nos últimos quarenta anos. O setor saúde implementou portarias que gradualmente incorporaram parâmetros físico-químicos, organolépticos e microbiológicos de qualidade, muitos deles com valores de referência baseados nas diretrizes da Organização Mundial da Saúde (OMS). Os parâmetros de potabilidade saltaram de 36 em 1990 para 84 hoje. As ações descritas na legislação possuem caráter intersetorial, onde diferentes atores possuem atribuições complementares e específicas. (FREITAS et al., 2005; HELLER et al., 2008; LIBÂNIO, 2010; QUEIROZ, 2012). Essa preocupação foi materializada em instrumentos jurídicos como a Lei 9.433, de 1997 (Lei das Águas), Portaria 1469, de 2000 (aprovando o padrão de qualidade da água para consumo humano) e a Lei 11.445 de 2007 (Lei do Saneamento) (HELLER et al., 2008).

A cidade de Ouro Preto, com população próxima a 74 mil habitantes, tem sua bacia inserida no Quadrilátero Ferrífero, cujas características geológicas possibilitam a ocorrência natural de certos metais como ferro, manganês, alumínio e arsênio. Neste contexto, os sistemas de abastecimento de água devem apresentar estruturas de tratamento que consigam remover satisfatoriamente estes elementos para níveis inferiores ao máximo permitido. $O$ tratamento deve considerar os aspectos específicos da bacia hidrográfica à qual está inserido, garantindo assim a qualidade da água fornecida à população (FERREIRA et al., 2006). Desde 2005, o abastecimento público de água está sob a responsabilidade do Serviço Municipal de Água e Esgoto (SEMAE-OP), uma autarquia municipal.

Os metais e semimetais ocorrem naturalmente na crosta terrestre, em diferentes variações e concentrações, regulados pelas propriedades do elemento e pela influência de fatores ambientais. Cerca de trinta metais e semimetais são potencialmente tóxicos para os seres humanos (MORAIS et al., 2012). Diversos metais e outros elementos possuem valor máximo permitido (VMP) definido na Portaria 2.914 / 2011, dada a possibilidade de ocorrência em fontes de abastecimento de água e potenciais riscos à saúde humana. Estes estão incluídos nas tabelas de substâncias químicas (antimônio, arsênio, bário, cádmio, chumbo, cobre, cromo, mercúrio, selênio e níquel) e organolépticas (alumínio, ferro, manganês e zinco). 
Para a remoção de metais e outros elementos traço no tratamento de água, o processo de oxidação seguido por filtração é bastante utilizado, respeitando-se a questão do viés econômico, a capacidade das instalações de tratamento, o pH da água e a presença de outros contaminantes na água. A presença de matéria orgânica natural, particularmente substâncias húmicas, aumenta a associação entre metais e óxidos que formam complexos e reduzem os íons metálicos livres. Processos de adsorção e troca iônica, préoxidação, ultrafiltração/osmose reversa na remoção destes elementos em água também podem ser utilizados. No entanto, o tratamento convencional ainda é o mais utilizado. No caso da cidade de Ouro Preto, uma importante contribuição foi feita por Pires et al. (2015), ao introduzir procedimentos complementares ao tratamento convencional em estações de tratamento de água.

Assim, este trabalho tem como objetivo apresentar resultados de análises de concentrações de manganês, ferro, cobre, zinco, arsênio, cromo e chumbo em amostras de dez sistemas de abastecimento de água e soluções alternativas coletivas alternativas do município de Ouro Preto/MG, durante a estação seca e chuvosa, observando os parâmetros estabelecidos pela classe 2 da Resolução CONAMA 357/2005 (água bruta) e pela Portaria 2.914 / 2011 (água para tratada).

\section{MATERIAIS E MÉTODOS}

As coletas foram realizadas em duas campanhas nos pontos de chegada de água bruta e na saída de água tratada de seis ETAs, e nos locais de captação e pontos de consumo das soluções alternativas coletivas, em momentos distintos: na estação seca e na estação chuvosa do ano de 2013 (junho e setembro). Estes períodos distintos se justificam pela dinâmica de composição química presente na água, por meio do carreamento de sedimentos e diferente volume de água nestes cursos hídricos, o que pode acarretar em diferentes concentrações de compostos químicos, como no caso dos metais em questão.

As coletas de água foram realizadas em dez sistemas de abastecimento de água com captação superficial. Os pontos de coleta escolhidos foram os locais de chegada de água bruta e de saída de tratamento. No caso dos sistemas de abastecimento com tratamento simplificado, no ponto de captação de água bruta e em um ponto do sistema de distribuição (Unidades Básicas de Saúde ou ponto do sistema de distribuição próximo à saída do reservatório). As coletas foram realizadas nas seis Estações de Tratamento de Água do município e também em quatro sistemas com tratamento simplificado (Figura 1). Durante as duas campanhas, foram coletados $50 \mathrm{~mL}$ em cada local, e em seguida foram encaminhadas ao Laboratório de Caracterização Molecular e Espectrometria de Massas (LCMEM-UFOP) para preparo e análise, conforme ilustrado na Figura 2.

A técnica de Fluorescência de Raio-X por Reflexão Total (TXRF) consiste em um importante método para rastreamento e análise de elementos traço. Inicialmente aplicada à indústria de semicondutores, nos últimos anos tem sido bastante utilizada em análises ambientais de solo e água, apresentando vantagens em razão do tamanho reduzido dos aparelhos mais recentes, além do baixo consumo da amostra, capacidade de análise multielementar simultânea e calibração simplificada. Além disto, pode-se identificar como vantagem do TXRF o baixo limite de detecção de determinados elementos, chegando a atingir limite de detecção de 
partes por bilhão, para o arsênio, como exemplo. A partir destas características, a aplicação do método tornou-se bastante utilizada, sobretudo a partir da década de 1990 (BORGUESE et al., 2015; MARGUÍ et al., 2014).

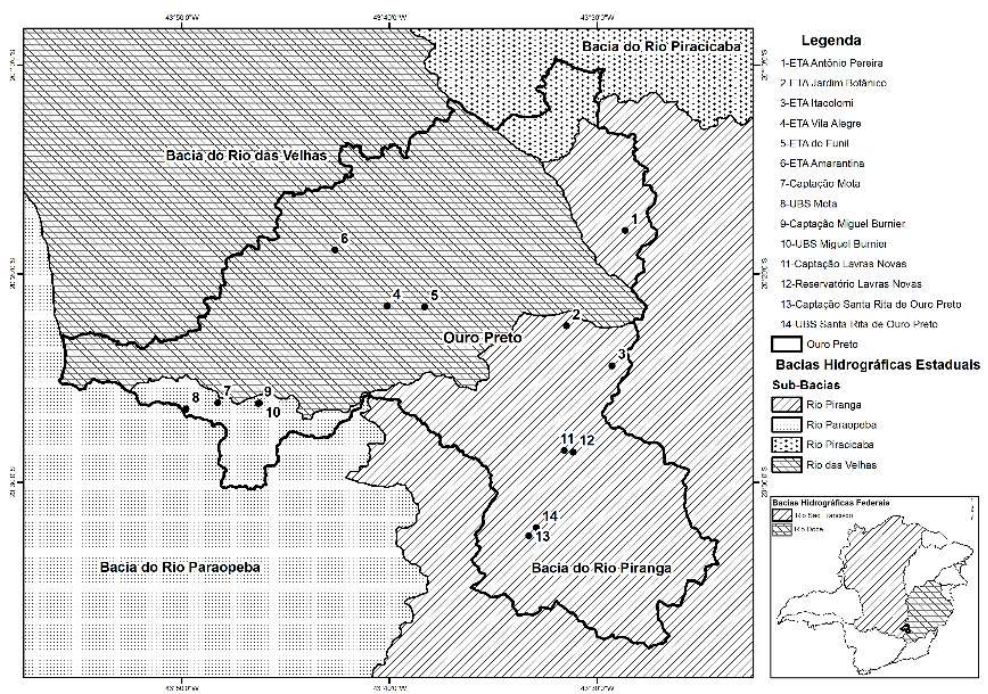

Figura 1: locais de coleta e suas respectivas bacias hidrográficas.

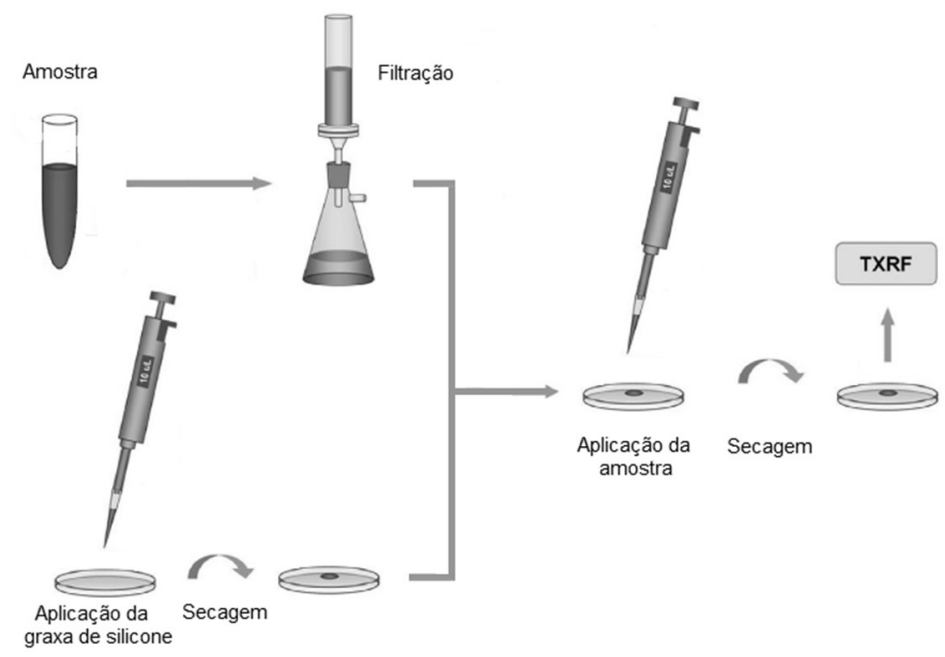

Figura 2: etapas de preparo da amostra. Fonte: Adaptado de Marguí et al. (2014).

\section{RESULTADOS}

Os resultados obtidos estão resumidos e descritos nas tabelas 1 e 2 .

Tabela 1: Resultados das análises de água bruta $\left(\mu \mathrm{g} \cdot \mathrm{L}^{-1}\right)$.

\begin{tabular}{|c|c|c|c|c|c|c|c|c|c|c|c|c|c|c|}
\hline \multirow[b]{2}{*}{ Período } & \multicolumn{2}{|l|}{$M n$} & \multicolumn{2}{|l|}{$\mathrm{Fe}$} & \multicolumn{2}{|l|}{$\mathrm{Cu}$} & \multicolumn{2}{|c|}{$\mathrm{Zn}$} & \multicolumn{2}{|l|}{ As } & \multicolumn{2}{|l|}{$\mathrm{Cr}$} & \multicolumn{2}{|l|}{$\mathrm{Pb}$} \\
\hline & Seco & Chuv. & Seco & Chuv. & Seco & Chuv & Seco & Chuv & Seco & Chuv & $\begin{array}{l}\text { Sec } \\
0\end{array}$ & Chuv & Seco & Chuv \\
\hline VMP* & \multicolumn{2}{|l|}{100} & \multicolumn{2}{|l|}{300} & \multicolumn{2}{|l|}{9} & \multicolumn{2}{|l|}{180} & \multicolumn{2}{|l|}{10} & \multicolumn{2}{|l|}{50} & \multicolumn{2}{|l|}{10} \\
\hline $\begin{array}{l}\text { Amarantin } \\
\text { a. }\end{array}$ & $\begin{array}{l}109 \\
9\end{array}$ & 131,8 & $\begin{array}{l}2.027, \\
4\end{array}$ & $\begin{array}{l}1.906, \\
8\end{array}$ & $\begin{array}{l}290, \\
8\end{array}$ & 4 & $\begin{array}{l}272, \\
2\end{array}$ & 39,9 & 8,06 & 5,7 & $<7$ & $<10$ & 10 & $<3$ \\
\hline $\begin{array}{l}\text { Antônio } \\
\text { Pereira }\end{array}$ & 25,3 & 27,4 & $<5,2$ & 33,5 & $<3,2$ & $<4,9$ & $\begin{array}{l}141, \\
7\end{array}$ & $\begin{array}{l}316 \\
8\end{array}$ & $<2,1$ & $<3,3$ & $\begin{array}{l}<8, \\
2\end{array}$ & 20,8 & $<3$ & $<4$ \\
\hline Funil & $\begin{array}{l}136, \\
5\end{array}$ & 239 & 164,4 & 660,8 & $<2$ & 4,71 & $\begin{array}{l}134, \\
7\end{array}$ & 49,3 & $<1,5$ & 14 & $\begin{array}{l}<5, \\
8\end{array}$ & 9,6 & $<2$ & $<3$ \\
\hline Itacolomi & $<4,1$ & $<7$ & 107,8 & 43,4 & $<2$ & $<3$ & $\begin{array}{l}203, \\
1\end{array}$ & $<2,7$ & 3,97 & $<2$ & $\begin{array}{l}<5, \\
1\end{array}$ & $<8$ & $<2$ & $<3$ \\
\hline
\end{tabular}




\begin{tabular}{|c|c|c|c|c|c|c|c|c|c|c|c|c|c|c|}
\hline $\begin{array}{l}\text { Jardim } \\
\text { Botânico }\end{array}$ & $<6,7$ & $<5,2$ & 124,1 & 56,9 & $<3,2$ & 3,41 & $\begin{array}{l}258, \\
8\end{array}$ & $<2$ & $<2,1$ & $\begin{array}{l}<1,5 \\
9\end{array}$ & $\begin{array}{l}<8 \\
3\end{array}$ & $<6,3$ & $<2,7$ & $<2$ \\
\hline Vila Alegre & $\begin{array}{l}125 \\
3\end{array}$ & $\begin{array}{l}1.179, \\
2\end{array}$ & 405,4 & $\begin{array}{l}1.479, \\
8\end{array}$ & $\begin{array}{l}414, \\
1\end{array}$ & $<2,6$ & $\begin{array}{l}348, \\
7\end{array}$ & $\begin{array}{l}145, \\
5\end{array}$ & 10,6 & 8,56 & $<15$ & $<7,4$ & 7,8 & $<2$ \\
\hline $\begin{array}{l}\text { Lavras } \\
\text { Novas } \\
\end{array}$ & 14,4 & 31,7 & 542,7 & 439,2 & $<2,3$ & $\begin{array}{l}<1,7 \\
1 \\
\end{array}$ & $\begin{array}{l}121, \\
5\end{array}$ & $<1,5$ & $\begin{array}{l}<1,5 \\
2 \\
\end{array}$ & 6,91 & $\begin{array}{l}<6, \\
1\end{array}$ & $<4,6$ & $<2$ & $<1$ \\
\hline $\begin{array}{l}\text { Miguel } \\
\text { Burnier }\end{array}$ & $<2,4$ & $<8,7$ & 25 & 17,9 & $<1,2$ & $<4$ & $\begin{array}{l}105, \\
8\end{array}$ & $<3,5$ & $<0,8$ & $<2,6$ & $<3$ & $\begin{array}{l}<10, \\
6\end{array}$ & $<1$ & $<3,3$ \\
\hline Mota & 33,9 & 66,9 & 36,6 & 179,3 & 67,2 & 5,91 & 49,6 & $\begin{array}{l}112, \\
6\end{array}$ & $\begin{array}{l}<0,8 \\
6\end{array}$ & 1,8 & $\begin{array}{l}<3 \\
3\end{array}$ & $<6,7$ & $\begin{array}{l}<1,0 \\
9\end{array}$ & $<2$ \\
\hline Santa Rita & 80,4 & 63,7 & 782,4 & $\begin{array}{l}4.383, \\
8\end{array}$ & $<5$ & $<2,7$ & $\begin{array}{l}286, \\
5\end{array}$ & $<2,3$ & 7,2 & 7,36 & $<14$ & $<7,5$ & $<4$ & 2,5 \\
\hline
\end{tabular}

Tabela 2: resultados de análises de água tratada $\left(\mu \mathrm{g} \cdot \mathrm{L}^{-1}\right)$.

\begin{tabular}{|c|c|c|c|c|c|c|c|c|c|c|c|c|c|c|}
\hline \multirow[b]{2}{*}{ Período } & \multicolumn{2}{|l|}{$M n$} & \multicolumn{2}{|l|}{$\mathrm{Fe}$} & \multicolumn{2}{|l|}{$\mathrm{Cu}$} & \multicolumn{2}{|l|}{$\mathrm{Zn}$} & \multicolumn{2}{|l|}{ As } & \multicolumn{2}{|l|}{$\mathrm{Cr}$} & \multicolumn{2}{|l|}{$\mathrm{Pb}$} \\
\hline & $\begin{array}{l}\mathrm{Sec} \\
\mathrm{o}\end{array}$ & Chuv & Seco & $\begin{array}{l}\text { Chuv } \\
\end{array}$ & Seco & Chuv & Seco & Chuv & Seco & Chuv & $\begin{array}{l}\mathrm{Sec} \\
\mathrm{O}\end{array}$ & Chuv & $\begin{array}{l}\mathrm{Sec} \\
\mathrm{o}\end{array}$ & $\begin{array}{l}\text { Chuv } \\
\end{array}$ \\
\hline VMP* & \multicolumn{2}{|l|}{100} & \multicolumn{2}{|l|}{300} & \multicolumn{2}{|l|}{2000} & \multicolumn{2}{|l|}{5000} & \multicolumn{2}{|l|}{10} & \multicolumn{2}{|l|}{50} & \multicolumn{2}{|l|}{10} \\
\hline $\begin{array}{l}\text { Amarantin } \\
\text { a }\end{array}$ & $<10$ & $<9$ & 66,1 & 98,2 & 5,6 & 6,91 & $\begin{array}{l}324 \\
3\end{array}$ & $\begin{array}{l}273, \\
6\end{array}$ & $<2,9$ & 5,2 & 12 & $<11$ & $<4$ & $<3$ \\
\hline $\begin{array}{l}\text { Antônio } \\
\text { Pereira }\end{array}$ & 36,1 & 15,9 & 16,8 & 28,2 & $\begin{array}{l}207, \\
9\end{array}$ & $<1,64$ & $\begin{array}{l}178, \\
3\end{array}$ & 38,9 & 2,57 & $<1,1$ & $<4,7$ & $<4,3$ & 3,59 & $<1$ \\
\hline Funil & $<5,3$ & $<5$ & 16,3 & 15,1 & $<2,6$ & $<2,4$ & $\begin{array}{l}195, \\
7\end{array}$ & $<2,1$ & $<1,7$ & $<1,54$ & $<6,6$ & $<6,2$ & $<2$ & $<2$ \\
\hline Itacolomi & $<11$ & $<5,2$ & $<8,3$ & 68,6 & $<5,2$ & $<2,4$ & 14,3 & $\begin{array}{l}117, \\
2\end{array}$ & $<3,5$ & $<1,6$ & $<14$ & $<6,4$ & $<4,5$ & 2,7 \\
\hline $\begin{array}{l}\text { Jardim } \\
\text { Botânico }\end{array}$ & $<9$ & 5,5 & 80,1 & 26,9 & $<4,3$ & $<2,5$ & $\begin{array}{l}189 \\
4 \\
\end{array}$ & $<2,3$ & $<2,9$ & $<1,7$ & $<11$ & $<7$ & $<4$ & $<2$ \\
\hline Vila Alegre & $<5,4$ & 10 & 28,5 & $<2,2$ & $<2,6$ & $<1,3$ & $\begin{array}{l}167, \\
4\end{array}$ & $<1,16$ & $<1,7$ & 2,89 & $<6,7$ & $<3,5$ & $<2$ & $<1$ \\
\hline $\begin{array}{l}\text { Lavras } \\
\text { Novas }\end{array}$ & 9,3 & 22,7 & $\begin{array}{l}176, \\
4\end{array}$ & $\begin{array}{l}709 \\
8\end{array}$ & 25 & $<1,71$ & $\begin{array}{l}242 \\
4\end{array}$ & $<1,5$ & $<1,9$ & 5,04 & $<7$ & $<4,6$ & $<2$ & $<1,45$ \\
\hline $\begin{array}{l}\text { Miguel } \\
\text { Burnier }\end{array}$ & 7 & $<6,9$ & 29,4 & 56,8 & 3,2 & $<3,3$ & $\begin{array}{l}173, \\
1\end{array}$ & $<2,9$ & 2,1 & 3 & 8 & 15 & 3 & $<3$ \\
\hline Mota & 38,6 & $\begin{array}{l}133, \\
4\end{array}$ & 63,6 & $\begin{array}{l}243, \\
7\end{array}$ & 1,83 & $<3,4$ & 53 & 10 & $<1,2$ & $<2,2$ & $<4,7$ & $<9$ & $<2$ & $<3$ \\
\hline Santa Rita & 45 & $<7,7$ & $\begin{array}{l}771, \\
6\end{array}$ & $\begin{array}{l}253 \\
6 \\
\end{array}$ & 3,2 & $<3,5$ & $\begin{array}{l}123 \\
7 \\
\end{array}$ & $<3,0$ & $\begin{array}{l}<1,7 \\
6\end{array}$ & 6,0 & $<6,8$ & $<9$ & $<2$ & 4,5 \\
\hline
\end{tabular}

*VMP: Valores Máximos Permitidos pela Portaria 2.914/2011.

\section{DISCUSSÃO}

Este trabalho identificou a presença dos elementos analisados na maioria das amostras, sendo encontrados níveis acima dos especificados para manganês, ferro e arsênio na água bruta, tomando como referência os valores da Portaria 2.914/2011. Para as amostras de água bruta, foram considerados os valores da classe 2 da Resolução Conama 357, que correspondem ao enquadramento dos cursos hídricos em questão. Concentrações de manganês acima do permitido para água bruta foram identificadas nas ETAs de Amarantina, Funil e Vila Alegre, em ambas as coletas. Nenhuma amostra de água tratada apresentou resultado acima do permitido para cobre, zinco, arsênio, cromo e chumbo. Para águas não tratadas foram encontradas quantidades significativamente maiores de resultados acima do máximo permitido, sugerindo eficácia dos mecanismos de tratamento na remoção destes. 
Destaca-se a concentração de manganês na ETA Vila Alegre, na coleta de água bruta do período chuvoso, com valor onze vezes acima do máximo permitido pela Portaria 2.914/2011 e também acima dos valores de referência da Resolução CONAMA 357/2005 para a classe 2, considerando-se o enquadramento do corpo hídrico. No entanto, quando analisadas as amostras de água tratada, o conjunto de estruturas de tratamento reduziu a concentração para valores inferiores ao máximo permitido pela Portaria 2.914/2011.

Durante a estação chuvosa normalmente ocorre um aumento da turbidez, que introduz uma quantidade significativa de sólidos em suspensão nos cursos d'água. Com isto, pode ocorrer uma maior concentração de metais e outras substâncias em razão do seu carreamento das margens e áreas de drenagem para os cursos hídricos. Como trata-se de região com histórico de mineração, a maior concentração destas sustâncias pode ser associada à atividade de extração mineral (artesanal ou industrial) (GUIMARÃES-SILVA et al., 2007).

Já em relação à água tratada, apenas uma amostra apresentou valor de manganês superior ao máximo permitido (Mota, período chuvoso). Esta amostra apontou concentração 30\% acima do máximo permitido. O manancial em questão se situa próximo a sites de mineração, o que pode interferir na concentração desta substância. Além disto, a única etapa de tratamento de água deste sistema consiste na desinfecção com cloro, onde pode ocorrer a oxidação deste elemento. Para o parâmetro ferro, apenas uma amostra (Santa Rita, período seco) apresentou valor acima do permitido. Assim como em Mota, a única etapa de tratamento em Santa Rita é a desinfecção com cloro.

Todos os resultados de análise das amostras de água tratada realizadas em sistemas com tratamento convencional apresentaram resultados satisfatórios, considerando a Portaria 2914/2011. Isto pode ser explicado pelo conjunto de etapas que compõem o tratamento convencional: mistura rápida, floculação, decantação, filtração e desinfecção, que em conjunto são capazes de reduzir significativamente os níveis destes elementos na água tratada (HELLER et al., 2006; LIBÂNIO, 2010).

Neste sentido, o trabalho realizado por Pires et al. (2015) contribuiu para a otimização das estruturas de tratamento convencional do município, uma vez que buscou reduzir a quantidade destes elementos na água tratada. Ao realizar ensaios de otimização do tratamento por clarificação para remoção de turbidez com Policloreto de Alumínio (PAC), além da implantação da etapa de pré-oxidação por meio da aplicação de Hipoclorito de Sódio em ETAs do município, conseguiu-se uma relevante redução de arsênio e manganês na água tratada.

Comportamento diferente apresentam os resultados relativos ao ferro nas amostras de água bruta. As duas coletas de água bruta de Amarantina, Lavras Novas, Santa Rita e Vila Alegre apresentaram valores superiores ao máximo permitido, enquanto uma das amostras de Funil também apresentou valor acima do permitido. Em relação às demais análises de água bruta, destaca-se a presença de arsênio acima do VMP na captação da ETA Vila Alegre na coleta do período chuvoso e na captação da ETA do Funil, no período chuvoso, ambas na microbacia do Rio Maracujá, em Cachoeira do Campo. Como se trata de elemento traço de grande risco à saúde em baixas concentrações, cabe destacar a importância de seu monitoramento constante. 
Os trabalhos de Borba et al. (2004), Varejão et al. (2011), Gonçalves et al. (2013) e Pires et al. (2015) identificaram presença de arsênio em águas superficiais e subterrâneas do município de Ouro Preto acima do VMP, considerando-se a Portaria 2914/2011 (VMP similar à classe 2 da Resolução CONAMA 357). Em 2007, o trabalho de Guimarães-Silva et al. encontrou resultados elevados de concentração de manganês no distrito de Rodrigo Silva.

Destaca-se que nenhuma amostra de água tratada analisada para este trabalho apresentou resultado superior ao máximo permitido para arsênio. Por outro lado, as amostras de água tratada apresentaram resultados abaixo do VMP para ferro em 19 das 20 amostras. A exceção foi a de água tratada do período chuvoso do distrito de Santa Rita de Ouro Preto, com resultado $150 \%$ maior do que o VMP. O fato de o sistema utilizar-se apenas da etapa de desinfecção no tratamento pode ser apontado como um elemento que substancia a hipótese da ineficiência do tratamento neste contexto. No entanto, é importante ressaltar a importância da análise de água bruta ao observar que ocorre consumo de água bruta por uma parcela da população local.

\section{CONCLUSÕES}

As análises de água bruta apontaram valores acima do permitido para ferro (9), manganês (6), cobre (3), zinco (6) e arsênio (2) considerando a classe 2 da Resolução CONAMA 357/2005, enquanto houve nas análises de água tratada valores acima do permitido apenas para ferro (1) e manganês (1), considerada a Portaria 2.914/2011. Não foi identificada a presença de arsênio acima do VMP nas análises de água tratada. Amostras de água tratada em sistemas simplificados apresentaram resultado insatisfatório, nas localidades de Santa Rita de Ouro Preto, para ferro (1), e Mota, para manganês (1). Por outro lado, todas as análises de água tratada oriundas de ETAs apresentaram resultado dentro dos parâmetros da Portaria 2.914.

Recomenda-se o monitoramento constante da qualidade da água dos sistemas de abastecimento de água do município, identificando a ocorrência destes e de outros elementos e substâncias elencadas na portaria. Caso estes elementos sejam encontrados em valores superiores ao máximo permitido, será necessária a busca por métodos de tratamento mais eficientes em sua remoção, visando assegurar à população o fornecimento de água que atenda aos parâmetros de potabilidade estabelecidos. Além disto, destaca-se a necessidade do trabalho de educação em saúde e monitoramento clínico junto à população que consome água bruta em locais com presença destes elementos com valores acima de parâmetros seguros à saúde.

\section{REFERÊNCIAS}

BORBA, R. P.; FIGUEIREDO, B. R.; CAVALCANTI, J. A.. Arsênio na água subterrânea em Ouro Preto e Mariana, Quadrilátero Ferrífero (MG). REM: Revista da Escola de Minas, Ouro Preto, v.57, n.1, p.45-51, 2004. DOI:

http://dx.doi.org/10.1590/S0370-44672004000100009

BORGUESE, L.; BILO, F.; DALIPI, R.; BONTEMPI, E.; DEPERO, L. E.. Total reflection $X$-ray fluorescence as a tool for food screening. Spectrochimica Acta Part B: Atomic
Spectroscopy, v.113 p.1-15, 2015. DOI: https://doi.org/10.1016/j.sab.2015.08.001

BRASIL. Resolução CONAMA n.357: Dispõe sobre a classificação dos corpos de água e diretrizes ambientais para o seu enquadramento, bem como estabelece as condições e padrões de lançamento de efluentes, e dá outras providências. Brasília: DOU, 2005. 
BRASIL. Portaria 2.914: Dispõe sobre os procedimentos de controle e de vigilância da qualidade da água para consumo humano e seu padrão de potabilidade. Brasília: DOU, 2011.

FERREIRA, A. C. S.; PÁDUA, V. L.. Qualidade da água para consumo humano. In: HELLER, L., PÁDUA, V. L..

Abastecimento de água para consumo humano. Belo Horizonte: UFMG, 2006.

FREITAS, M. F., FREITAS, C. M.. A vigilância da qualidade da água para consumo humano: desafios e perspectivas para o Sistema Único de Saúde. Revista Ciência e Saúde Coletiva, v.10 n.4, 2005. DOI: http://dx.doi.org/10.1590/S141381232005000400022

GONÇALVES, J. A. C.; LENA, J. C.. Avaliação de risco à saúde humana por contaminação natural de arsênio nas águas subterrâneas e nos solos da área urbana de Ouro Preto (MG). Revista do Instituto de Geociências - USP Geol, São Paulo, v.13, n.2, p.114-148, 2013. DOI: http://dx.doi.org/10.5327/Z1519-874X2013000200008

GUIMARÃES-SILVA, A. K.; MACHADO, D. A.; NALINI JR.; H. A.; LENA, J. C.. A qualidade das águas na região dos garimpos de topázio imperial na sub-bacia do rio da Ponte, Ouro PretoMG. Revista Escola de Minas, Ouro Preto, v.60, n.4, p.603611, 2007. DOI: http://dx.doi.org/10.1590/S037044672007000400005

HELLER, L., PÁDUA, V. L.. Abastecimento de água para consumo humano. Belo Horizonte: UFMG, 2006.

LIBÂNIO, M.. Fundamentos de qualidade e tratamento de água. Campinas: Átomo, 2010.

MARGUÍ, E.; ZAWISZA, B.; SITKO, R.. Trace and ultratrace analysis of liquid samples by $\mathrm{X}$-ray fluorescence spectrometry. Trends in Analytical Chemistry, v.53, p.73-83, 2014. DOI: https://doi.org/10.1016/i.trac.2013.09.009

MORAIS, S.; GARCIA, F.; PEREIRA, M. L.. Heavy Metals and Human Health. In: OOSTHUIZEN, J.. Environmental Health Emerging Issues and Practice. Rijeka: InTech, 2012.

PIRES, V. G. R; LIMA, D. R. S; AQUINO, S. F.; LIBÂNIO, M.. Arsenic and Manganese Removal From Water by Chlorine Oxidation Followed by Clarification. Brazilian Journal of Chemical Engineering, v.32, n.2, p.409-419, 2015. DOI: http://dx.doi.org/10.1590/0104-6632.20150322s00003564

QUEIROZ, A. C. L., CARDOSO, L. S. M., SILVA, S. C. F., HELLER L., CAIRNCROSS, S.. Programa Nacional de Vigilância em Saúde Ambiental Relacionada à Qualidade da Água para Consumo Humano (Vigiagua): lacunas entre a formulação do programa e sua implantação na instância municipal. Revista Saúde e Sociedade, São Paulo, v.21, n.2, 2012. DOI: http://dx.doi.org/10.1590/S0104-12902012000200019

SANTOS, J. S.; SANTOS, M. L. P.; OLIVEIRA, E.. Estudo da mobilização de metais e elementos traços em ambientes aquáticos do semiárido brasileiro aplicando análises de componentes principais. Química Nova, v.31, n.5, p.11071111,2008

VAREJÃO, E. V. V.; BELLATO, C. R.; FONTES, M. P. F.; MELLO, J. W. V.. Arsenic and trace metals in river water and sediments from the southeast portion of the Iron Quadrangle, Brazil. Environmental monitoring and assessment, v.172, n.1, p.631-642, 2011. DOI: https://doi.org/10.1007/s10661-010-1361-3

VON SPERLING, M.. Introdução a qualidade das águas e ao tratamento de esgotos. 3 ed. Belo Horizonte: Departamento de Engenharia Sanitária e Ambiental; Universidade Federal de Minas Gerais, 2005.

A CBPC - Companhia Brasileira de Produção Científica (CNPJ: 11.221.422/0001-03) detém os direitos materiais desta publicação. Os direitos referem-se à publicação do trabalho em qualquer parte do mundo, incluindo os direitos às renovações, expansões e disseminações da contribuição, bem como outros direitos subsidiários. Todos os trabalhos publicados eletronicamente poderão posteriormente ser publicados em coletâneas impressas sob coordenação da Sustenere Publishing, da Companhia Brasileira de Produção Científica e seus parceiros autorizados. Os (as) autores (as) preservam os direitos autorais, mas não têm permissão para a publicação da contribuição em outro meio, impresso ou digital, em português ou em tradução. 